\title{
INMUNOHISTOQUÍMICA EN PATOLOGÍA DE MAMA. DIFERENCIACIÓN DE LESIONES COMPLEJAS BENIGNAS Y MALIGNAS DE MAMA: UN REPORTE DE CASO Y REVISIÓN DE LA LITERATURA
}

\section{Immunohistochemistry in breast pathology. Differentiating complex benign and malign breast lesions: a case report and literature review}

Omaira del Socorro Herrera-Arias, M.D.*, Jaime Díaz-Cardona, M.D. **

Recibido: noviembre 8/10 - Aceptado: septiembre 5/11

\section{RESUMEN}

Objetivo: revisar las bases histológicas y los criterios diagnósticos diferenciales de la aplicación de la inmunohistoquímica en la patología mamaria. Caso clínico: se presenta el caso de una paciente con lesión mamaria esclerosante compleja, tipo adenosis microglandular (MGA), que requirió el uso de inmunohistoquímica como técnica auxiliar para diferenciar la lesión de un adenocarcinoma de mama in situ, y definir así el tratamiento.

Materiales y métodos: se realizó una búsqueda de la información en las bases de datos Medline vía PubMed, SciELO y en libros de la especialidad. Se reportaron 25 títulos, 12 correspondían con el tema: 4 reportes de caso, 1 con revisión de la literatura, una carta al editor y 7 artículos relacionados de forma más general con el tema.

Conclusión: la inmunohistoquímica permite hacer el diagnóstico diferencial de las lesiones mamarias

* Médico y Cirujano, Universidad de Antioquia. Residente II año Ginecología y Obstetricia, Universidad CES. Medellín (Colombia). Correo electrónico: omairacarolinaha@yahoo.es

** Médico y Cirujano, Universidad de Caldas. Especialista en Ginecología y Obstetricia, Universidad de Caldas. Ginecólogo Oncólogo, Universidad Javeriana, Instituto Nacional de Cancerología. Especialista Auditoría Médica, Medellín (Colombia). Correo electrónico: jaime.diaz30@yahoo.com.co complejas, como la adenosis esclerosante y el cáncer de mama in situ o microinvasor.

Palabras clave: inmunohistoquímica en mama, adenosis esclerosante, lesiones de mama.

\section{SUMMARY}

Objective: reviewing the histological basis and differential diagnostic criteria for applying immunohistochemistry in breast pathology.

Clinical case: the case of a patient suffering from complex sclerosing lesion (CSL) of the breast, microglandular adenosis (MGA) pattern is presented; she required immunohistochemistry as an auxiliary technique for differentiating an adenocarcinoma-related breast lesion in situ and thus defining its treatment.

Materials and methods: a search was made of the pertinent information in Medline databases via PubMed, SciELO and in books on the specialty; 25 titles were reported, 12 of them corresponding to the immediate topic: 4 were case reports with a literature review and one was a letter to the editor. 7 articles dealt with the topic in a more general way. Conclusion: immunohistochemistry led to the differential diagnosis of complex breast lesions such 
as sclerosing adenosis, and in situ or microinvase breast cancer.

Key words: imunohistochemistry of the breast, sclerosing adenosis, breast lesions.

\section{INTRODUCCIÓN}

En la patología de mama es fundamental hacer un diagnóstico diferencial entre la adenosis y el carcinoma de mama. La adenosis es la enfermedad de la mama más frecuente que hace parte del complejo grupo de enfermedades con cambios fibroquísticos benignos. ${ }^{1}$ Descrita por FooteStewart en 1949, esta entidad presenta un cuadro clínico más frecuente en mujeres premenopáusicas; se manifiesta, en la mayoría de los casos, como una lesión nodular no dolorosa y variable, aislada o asociada a fibroadenomas u otros, además, puede presentar en la imagenología microcalcificaciones que caracterizan una adenosis esclerosante o una lesión espiculada. ${ }^{2}$

La adenosis se caracteriza histológicamente por tener un patrón proliferativo derivado de la unidad terminal dúctu lobular, con proliferación moderada de las células mioepiteliales, lo que aumenta el número de acinos cercano al 50\%, especialmente en la adenosis esclerosante. Esta entidad se asocia a distorsión y elongación, con o sin obliteración, de las glándulas y túbulos, sumado a un estroma esclerótico con microquistes, microcalcificaciones y simulaciones de tumores malignos. ${ }^{3,4}$ La arquitectura lobulillar se conserva, pero puede presentarse extensión grasa y perineural; además estas lesiones, con extensión elongada, tienen mayor riesgo de convertirse en lesiones malignas. ${ }^{5}$

La adenosis esclerosante se puede asociar y presentar con otras lesiones proliferativas benignas, como papiloma intraductal y/o esclerosante, lesión esclerosante compleja, fibroadenomas, entre otras, asimismo puede coexistir con lesiones invasoras o carcinoma in situ. ${ }^{6-8}$

$\mathrm{La}$ adenosis tiene varios patrones histológicos como son:
- Adenosis esclerosante.

- Adenosis tumoral.

- Adenosis apocrina.

- Adenosis tubular.

- Adenosis mioepitelial.

- Adenosis microglandular.

- Cicatriz radial/lesión esclerosa compleja.

La adenosis esclerosante, la adenosis microglandular típica (MGA), y especialmente la adenosis microglandular atípica (AMGA) son las lesiones proliferativas sin atipias (grupo adenosissegundo de la clasificación histopatológica de Page y Dupont) con mayor riesgo para el desarrollo de carcinoma de mama. ${ }^{9-13}$ La identificación de la capa de células mioepiteliales en mama es fundamental para el abordaje del diagnóstico histológico, ya que permite mayor aproximación diagnóstica que la ofrecida por la identificación de la membrana basal. La identificación de estas capas se realiza con la aplicación de los marcadores de inmunohistoquímica, seleccionando los más sensibles y específicos. ${ }^{14}$

La inmunohistoquímica permite completar el abordaje diagnóstico de lesiones mamarias, facilitando la diferenciación de lesiones benignas complejas y lesiones malignas de bajo o alto grado, diferenciación de lesiones in situ de lesiones invasivas, diferenciación de patrón tumoral, diagnóstico de lesiones metastásicas, y caracterización del ganglio centinela, entre otras. ${ }^{15}$ Es importante para el patólogo conocer los marcadores de inmunohistoquímica que se utilizan en el diagnóstico diferencial de la patología de seno, por lo que se presenta un caso en el que estos marcadores fueron útiles para hacer el diagnóstico definitivo, con el objetivo de revisar las bases histológicas y los criterios diagnósticos diferenciales de la aplicación de la inmunohistoquímica en la patología mamaria.

\section{REPORTE DE CASO}

Paciente de 48 años evaluada el día 27 de noviembre de 2009 en el hospital general de Medellín, centro de referencia de la región noroccidental 
de Colombia. Valorada en la consulta de ginecooncología por presentar, en la mamografía, lesión tipo nódulo con calcificaciones sospechosas de malignidad, reportadas como BIRADS 0/6. Se tomó biopsia que fue informada como hiperplasia ductal con atipias. A partir de la patología se sugiere la realización de inmunohistoquímica p63 y miosina de músculo liso. La paciente no presenta antecedentes de importancia para patología mamaria. En el examen físico se encontró, como hallazgo positivo en mama derecha, un nódulo de $3 \mathrm{~cm}$ con reacción desmoplásica y axila normal. Se realizó una nueva evaluación de la biopsia en donde se informó sospecha de carcinoma intraductal de grado nuclear bajo, pero el examen no fue concluyente. La paciente fue programada para una cuadrantectomía con congelación de márgenes y con posibilidad de mastectomía y/o vaciamiento axilar. La paciente fue conducida a cirugía el 8 de marzo de 2010, en donde se le realizó biopsia por congelación intraoperatoria, procedimiento que reportó carcinoma ductal in situ con compromiso del margen interno. Se procede a realizar la cuadrantectomía con ampliación del margen interno. El resultado de la patología fue reportado como hiperplasia florida con adenosis microglandular (MGA) y adenosis esclerosante frente a adenocarcinoma in situ. Sugieren inmunohistoquímica para realizar el diagnóstico diferencial. El resultado de la inmunohistoquímica evidenció: miosina de cadena pesada positiva en todas las formaciones tubulares; p63: positiva para células mioepiteliales, interpretado como: hallazgos a favor de lesión benigna de tipo adenosis esclerosante y adenosis tubular.

\section{MATERIALES Y MÉTODOS}

Con base en los términos immunohistochemistry in breast, sclerosing adenosis, myoepithelial cells, benign sclerosing lesions, ductal carcinoma in situ, se realizó una búsqueda de la información en las bases de datos Medline vía PubMed, SciELO, y en libros de la especialidad.

\section{RESULTADOS}

Se reportaron 25 títulos, 12 correspondían con el tema: 4 reportes de caso, 1 con revisión de la literatura, una carta al editor y 7 artículos relacionados de forma más general con el tema.

\section{DISCUSIÓN}

\section{Bases histológicas}

La inmunohistoquímica es una técnica que combina la histología con la inmunología, basada en la determinación de antígenos celulares (su presencia y localización microanatómica) a través del reconocimiento antígeno anticuerpo. ${ }^{16}$ Inicia su desarrollo desde el año 1940 cuando Coons utiliza la técnica de inmunofluorescencia en el análisis de tejidos; pero solo adquiere relevancia desde la década del 90 con el desarrollo de la patología molecular, y con la creación de múltiples técnicas y tinciones. ${ }^{17}$

Estos antígenos también son denominados marcadores. En la mama se encuentran diferentes tipos de marcadores usados en inmunohistoquímica que identifican las células luminales: citoqueratinas CK7, CK8, CK18, CK19; células basales: CK5/6, CK14, CK17; células mioepiteliales: CK5, CK14, CK17, SMA, SM, calponina, p63; y otros marcadores como la proteína s-100, CD10, caderina, receptores de estrógenos, progesterona, y HER2. ${ }^{17}$

\section{Criterios diagnósticos diferenciales}

En la patología de mama es fundamental la identificación de la capa de células mioepiteliales para el abordaje del diagnóstico histológico, mucho más que la identificación de la membrana basal y la capa de células epiteliales. Las lesiones benignas no invasivas y las lesiones malignas in situ de la mama presentan, en la histología, un patrón proliferativo de las capas de células epiteliales y mioepiteliales, acompañado de pleomorfismo y de malignidad en el segundo caso señalado; y en ambos tipos de lesiones se conserva la integridad, tanto de la capa de células mioepiteliales como de la membrana basal subyacente. ${ }^{18}$ Esta característica las hace positivas a la 
tinción inmunohistoquímica que utiliza marcadores dirigidos hacia las células mioepiteliales; entre ellos, marcadores de tipo citoplasmático como la cadena pesada de la miosina de músculo liso (SM-MHC), la actina del músculo liso (SMA), la calponina, y las citoqueratinas, además positivas ante marcadores de tipo nuclear como la proteína p63. Esta proteína es un factor de trascripción, miembro de la familia p53 y homóloga a ella, también tiene tinción positiva para células basales de reserva en la piel y próstata. ${ }^{15,17,19-22}$

Los marcadores citoplasmáticos SMA, SMMHC y la calponina se localizan en el citoplasma de las células mioepiteliales y en el citoplasma de otras células con inmunofenotipo similar, como las células del músculo liso de los vasos, células epiteliales producidas por tumores y en los miofibroblastos del estroma (con más frecuencia la SMA); lo que permite tinciones positivas y débilmente positivas generando falsos positivos para el diagnóstico de la capa de células mioepiteliales. En los casos dudosos se recomienda la técnica de la doble tinción: en la que se utilizan la prueba SM-MHC como marcador citoplasmático y la p63 como marcador nuclear que solo tiene tinción positiva en el núcleo de células mioepiteliales, y no tiene reacciones cruzadas con otras células del tejido mamario; mejorando su sensibilidad y especificidad. ${ }^{17} \mathrm{Si}$ persisten las dudas se aconseja complementar con la calponina. La técnica de la doble tinción permite una mayor aproximación diagnóstica en la diferenciación de lesiones benignas y lesiones malignas in situ de las lesiones invasivas. Una segunda opción de falsos positivos para células mioepiteliales se presenta cuando las células de un tumor invasivo se diferencian a células mioepiteliales, logrando una tinción positiva, como es el caso del adenocarcinoma escamoso de bajo grado, mioepitelioma maligno, carcinoma adenocístico. ${ }^{17-22}$

En las lesiones de tipo maligno, invasivas, hay ruptura de la capa de células mioepiteliales y de la membrana basal, por consiguiente, la tinción inmunohistoquímica es negativa, tanto para los marcadores citoplasmáticos como para el marcador nuclear p63. ${ }^{23,24}$ De esta manera, la inmunotinción negativa con marcadores de doble tinción, para células mioepiteliales, diagnostica lesión maligna invasiva de mama (ausencia de capa de células mioepiteliales); la única excepción es la adenosis microglandular (MGA), única condición benigna proliferativa que carece de capa de células mioepiteliales.

Por otra parte, se ha sugerido que en la biopsia por aspiración con aguja fina (BACAF), la utilización de la inmunohistoquímica específicamente del marcador nuclear p63, mejora la exactitud diagnóstica ya que está dirigido a los marcadores de los núcleos que se conservan en este tipo de muestra; puesto que los citoplasmas se alteran o pierden. Harton et $\mathrm{al}^{24}$ refieren un incremento de la sensibilidad y la especificidad del 90\% al 97\%, también reportado en la revisión realizada por Chaiwun et al. ${ }^{25,26}$

Por último, se ha descrito la utilidad de la inmunohistoquímica en la diferenciación de adenosis esclerosante y carcinoma ductal infiltrante. La adenosis esclerosante de mama presenta inmunotinción positiva para los marcadores de doble tinción: SMMHC (marcador citoplasmático) y p63 (marcador nuclear), y negativo para S-100, mientras que el carcinoma de mama invasivo presenta inmunotinción negativa para estos 2 marcadores y S-100. La adenosis microglandular (MGA) tiene tinción negativa por ausencia de la capa de células mioepiteliales, por ello simula una lesión maligna invasiva, y tanto la forma atípica AMGA como la típica se caracterizan por inmunotinción positiva para el marcador S-100 y las citoqueratinas (CK), asociados a receptores triple negativo: receptor de estrógeno (ER), receptor de progesterona (PR) y HER-2. ${ }^{27}$

\section{CONCLUSIÓN}

La inmunohistoquímica permite hacer el diagnóstico diferencial de las lesiones mamarias complejas, como la adenosis esclerosante y el cáncer de mama in situ o microinvasor. 


\section{REFERENCIAS}

1. Chen JH, Nalcioglu O, Su MY. Fibrocystic change of the breast presenting as a focal lesion mimicking breast cancer in MR imaging. J Magn Reson Imaging 2008;28:1499-505.

2. Gill HK, Ioffe OB, Berg WA. When is a diagnosis of sclerosing adenosis acceptable at core biopsy? Radiology 2003;228:50-7.

3. Chen JH, Nalcioglu O, Su MY. Fibrocystic change of the breast presenting as a focal lesion mimicking breast cancer in MR imaging. J Magn Reson Imaging 2008;28:1499-505.

4. Pojchamarnwiputh S, Muttarak M, Na-Chiangmai W, Chaiwun B. Benign breast lesions mimicking carcinoma at mammography. Singapore Med J 2007;48:958-68

5. Rosen PP. Rosen's breast pathology. 2nd ed. Philadelphia: Lippincott Williams \& Wilkins; 2001.

6. Meisner AL, Fekrazad MH, Royce ME. Breast disease: benign and malignant. Med Clin North Am 2008;92:1115-41.

7. Berek JS. Berek \& Novak's Gynecology, 14th ed. Philadelphia: Lippincott Williams \& Wilkins; 2006.

8. Santen RJ, Mansel R. Benign breast disorders. N Engl J Med 2005;353:275-85.

9. Geyer FC, Kushner YB, Lambros MB, Natrajan R, Mackay A, Tamber N, et al. Microglandular adenosis or microglandular adenoma? A molecular genetic analysis of a case associated with atypia and invasive carcinoma. Histopathology 2009;55:732-43.

10. Harmon M, Fuller B, Cooper K. Carcinoma arising in microglandular adenosis of the breast. Int J Surg Pathol 2001;9:344.

11. Shui R, Yang W. Invasive breast carcinoma arising in microglandular adenosis: a case report and review of the literature. Breast J 2009;15:653-6.

12. Resetkova E, Flanders DJ, Rosen PP. Ten-Year followup of mammary carcinoma arising in microglandular adenosis treated with breast conservation. Arch Pathol Lab Med 2003;127:77-80.

13. Visscher DW. Apocrine ductal carcinoma in situ involving sclerosing lesion with adenosis: report of a case. Arch Pathol Lab Med 2009;133:1817-21.

14. Fitzgibobons PL, Henson DE, Hutter RV. Bening breast changes and the risk for subsequent breast cancer: and update of the 1985 consensus statement. Cancer Committee of the College of American
Pathologists. Arch Pathol Lab Med 1998;122:1053-5.

15. Kalof AN, Tam D, Beatty B, Cooper K. Immunostaining patterns of myoepithelial cells in breast lesions: a comparison of CD10 and smooth muscle myosin heavy chain. J Clin Pathol 2004;57:625-9.

16. Yeh IT, Mies C. Application of immunohistochemistry to breast lesions. Arch Pathol Lab Med 2008;132:349-58.

17. Dabbs DJ. Diagnostic immunohistochemistry: theranostic and genomic applications. 3rd ed. Philadelphia: Saunders/Elsevier; 2010.

18. Hilson JB, Schnitt SJ, Collins LC. Phenotypic alterations in myoepithelial cells associated with benign sclerosing lesions of the breast. Am J Surg Pathol 2010;34:896-900.

19. Werling RW, Hwang H, Yaziji H, Gown AM. Immunohistochemical distinction of invasive from noninvasive breast lesions: a comparative study of p63 versus calponin and smooth muscle myosin heavy chain. Am J Surg Pathol 2003;27:82-90.

20. Boyd NF, Guo H, Martin LJ, Sun L, Stone J, Fishell E, et al. Mammographic density and the risk and detection of breast cancer. N Engl J Med 2007;3 56:227-36.

21. Yeh IT, Mies C. Application of immunohistochemistry to breast lesions. Arch Pathol Lab Med 2008;132:349-58.

22. Lervwill MF. Current practical applications of diagnostic immunohistochemistry in breast pathology. Am J Surg Pathol 2004;28:1076-91.

23. Tang P, Skinner KA, Hicks DG. Molecular classification of breast carcinomas by immunohistochemical analysis: are we ready? Diagn Mol Pathol 2009;18:125-32.

24. Harton AM, Wang HH, Schnitt SJ, Jacobs TW. p63 immunocytochemistry improves accuracy of diagnosis with fine-needle aspiration of the breast. Am J Clin Pathol 2007;128:80-5.

25. Chaiwun B, Thorner P. Fine needle aspiration for evaluation of breast masses. Curr Opin Obstet Gynecol 2007;19:48-55.

26. Berg WA, Sechtin AG, Marques H, Zhang Z. Cystic breast masses and the ACRIN 666 experience. Radiol Clin North Am 2010;48:931-87.

27. Shin SJ, Simpson PT, Da Silva L, Jayanthan J, Reid L, Lakhani SR, et al. Molecular evidence for progression of microglandular adenosis (MGA) to invasive carcinoma. Am J Surg Pathol 2009;33:496-504. 